\title{
Modeling species distributions by breaking the assumption of self-similarity
}

\author{
Cang Hui and Melodie A. McGeoch \\ C. Hui (chui@sun.ac.za) and M. A. McGeoch, Centre of Excellence for Invasion Biology, Dept of Conservation Ecology and \\ Entomology, Univ. of Stellenbosch, Private Bag X1, Matieland 7602, South Africa.
}

\begin{abstract}
Species distributions are commonly measured as the number of sites, or geographic grid cells occupied. These data may then be used to model species distributions and to examine patterns in both intraspecific and interspecific distributions. Harte et al. (1999) used a model based on a bisection rule and assuming self-similarity in species distributions to do so. However, this approach has also been criticized for several reasons. Here we show that the self-similarity in species distributions breaks down according to a power relationship with spatial scales, and we therefore adopt a power-scaling assumption for modeling species occupancy distributions. The outcomes of models based on these two assumptions (self-similar and power-scaling) have not previously been compared. Based on Harte's bisection method and an occupancy probability transition model under these two assumptions (self-similar and power-scaling), we compared the scaling pattern of occupancy (also known as the area-of-occupancy) and the spatial distribution of species. The two assumptions of species distribution lead to a relatively similar interspecific occupancy frequency distribution pattern, although the spatial distribution of individual species and the scaling pattern of occupancy differ significantly. The bimodality in occupancy frequency distributions that is common in species communities, is confirmed to a result for certain mathematical and statistical properties of the probability distribution of occupancy. The results thus demonstrate that the use of the bisection method in combination with a power-scaling assumption is more appropriate for modeling species distributions than the use of a self-similarity assumption, particularly at fine scales.
\end{abstract}

One of the most common ways in which species distributions are measured, particularly at scales smaller than the entire geographic range, is the number of sites or geographic grid squares that the species occupies (Gaston and Blackburn 2000). In assessments of interspecific patterns of species range sizes, these occupancy data are often expressed in the form of occupancy frequency distributions (McGeoch and Gaston 2002). The shape of these occupancy frequency distributions is commonly strongly right-skewed (dominant satellite mode sensu Hanski 1982), demonstrating that most species in a community have comparatively narrow ranges (Hanski 1982, Gaston 1994, 1996).

However, when compiled at scales much finer than species geographic ranges, bimodal occupancy frequency distributions are often equally common (McGeoch and Gaston 2002), i.e. with a mode in both the highest occupancy as well as lowest occupancy classes. Indeed, the occupancy frequency distribution has been shown to be highly scale-dependent (Wiens 1989, Gaston 1996, Blackburn and Gaston 1997, Cowley et al. 1999, Guo et al. 2000, He and Gaston 2000). For example, the number of satellite (low occupancy) species will decrease with grain but increase with extent or sample number; with the opposite being true for high occupancy species (core species, sensu Hanski 1982) (McGeoch and Gaston 2002). Nonetheless, McGeoch and Gaston (2002) categorized the shapes of the occupancy frequency distributions into eight forms, and demonstrated that empirical, satellitedominated bimodal occupancy frequency distributions are particularly common, i.e. most species being either rare or widely distributed, albeit largely at fine spatial scales.

Three basic models have been proposed to explain the bimodality found in occupancy frequency distributions. 
First, random sampling of individuals from either lognormal or log-series rank abundance distributions (where random choice of an individual from a given species was proportional to its frequency) may produce bimodal occupancy distributions (Nee et al. 1991, Papp and Izsák 1997). However, unimodal occupancy distributions may also be generated from such abundance distributions, and lognormal and logseries distributions have been shown roughly to fit abundance data for assemblages that have a wide range of species richness, total abundance, and occupancy distributions (McGeoch and Gaston 2002). This model is thus not particularly sensitive or informative as to the mechanisms generating bimodality in occupancy frequency distributions.

Second, bimodality may be generated by colonization-extinction metapopulation dynamics associated with a strong rescue effect (Hanski 1982, Hanski and Gyllenberg 1993). This model is appropriate to explain the range structure of a community that is influenced by metapopulation processes, such as dispersal and local extinction (Storch and Sizling 2002). However, it is not robust because the shape of the occupancy frequency distribution generated by this model is highly sensitive to species immigration and extinction parameters (Tokeshi 1992, Scheiner and Rey-Benayas 1997). The metapopulation model does also not explain scaledependence in the occupancy frequency distribution. Indeed, Van Rensburg et al. (2000) suggested that multiple mechanisms, including metapopulation processes and sampling artifacts, are likely to be necessary to explain the shape of occupancy distributions (also McGeoch and Gaston 2002).

The third model that describes bimodality in the occupancy frequency distribution is based on the scaling pattern of occupancy under a self-similar assumption (according to Harte et al. 1999) of species distributions (called the occupancy probability transition [OPT] model; Hui and McGeoch 2007a). The OPT model is based on Harte et al.'s (1999) bisection scheme (although not on their probability rule) and the recursion probability of occupancy at different scales. The latter is because species occupancy (or range size) is well known to be strongly affected by the scale at which it is measured (grain and extent; Kunin 1998, McGeoch and Gaston 2002, Hartley and Kunin 2003). The OPT model has been shown to support two empirical observations, i.e. (1) that bimodality is prevalent in interspecific occupancy frequency distributions and (2) that the number of satellite species in the distribution increases towards finer scales (Hui and McGeoch 2007a). The OPT model therefore demonstrates that the sample grain of a study, sampling adequacy, and the distribution of species saturation coefficients (a measure of the fractal dimensionality of a species distribution) in a community are together largely able to explain the patterns commonly found in empirical occupancy distributions.

However, there is general consensus that neither species distribution nor ecological processes are likely to have perfectly self-similar (scale-invariant) properties (Halley et al. 2004). Although the use of a selfsimilarity assumption (sensu Harte et al. 1999) when modeling species distributions has led to novel insights on community assemblage rules and macroecological patterns (Harte and Kinzig 1997, Harte et al. 1999, $2001,2005)$, the validity of a self-similar probability rule assumption for species distributions has been strongly criticized (Lennon et al. 2002, Maddux 2004, Ostling et al. 2004, Pueyo 2006).

Therefore, here we break the self-similar assumption in the OPT model and compare the outcomes of doing so with (1) those generated under self-similarity conditions (see also Hui and McGeoch 2007a), and (2) with empirical data. We do so to assess the consequences of breaking the self-similarity assumption in the OPT model for (1) the scaling pattern of occupancy and (2) the shape, and prevalence of bimodality in, occupancy frequency distributions. The approach to modelling interspecific species distribution patterns that we adopt here is different from Harte et al.'s (1999) much criticized probability rule. Instead, we allow the distribution of individual species to vary both between species (see also šizling and Storch 2004, Hui and McGeoch 2007a) and across scales, i.e. species do not have self-similar distributions. We then combine individual species distributions (each with different scaling properties) into community level occupancy frequency distributions.

\section{Model}

\section{Background}

To analyze the assemblage structure of a community when scaling down (i.e. with a decrease in grain), a process of landscape subdivision may be used (Harte et al. 1999, Plotkin et al. 2000, He and Legendre 2002). For example, Harte et al. (1999, 2005) examined the species-area relationship and the relative-abundance distribution using a bisection process along with a self-similar assumption for species distributions. Hui and McGeoch (2007a) extended the application of this approach to the examination of occupancy frequency distributions. As discussed above, here we aim to break the self-similarity assumption of species distribution in the occupancy probability transition model (OPT model, Hui and McGeoch 2007a) and test the effect of doing so on the scaling pattern of occupancy and on the shape of occupancy frequency distributions. 
If there is a species inhabiting a landscape unit with the area $A_{0} / 2^{i}\left(A_{0}\right.$ is the area of the original patch; after the ith bisection, the number of patches is $2^{\mathrm{i}}$ ), then there are two mutually exclusive states after the subdivision of the landscape unit into two. The one is that both subdivided units are occupied by the species; the other is that the species is located in only one of the two units. Let $\mathrm{p}_{\mathrm{i}}$ be the probability of the first state and $\mathrm{q}_{\mathrm{i}}$ that of the other. If we know the proportional occupancy is $\mathrm{O}_{\mathrm{i}}\left(\mathrm{O}_{\mathrm{i}}=\mathrm{j} / 2^{\mathrm{i}}\right.$ and $1 \leq \mathrm{j} \leq 2^{\mathrm{i}}$ after the ith bisection), the possible occupancy after bisection will be $\mathrm{O}_{\mathrm{i}+1}=(2 \mathrm{j}-\mathrm{k}) / 2^{\mathrm{i}+1}(0 \leq \mathrm{k} \leq \mathrm{j})$, with the minimal and maximal proportional occupancy $\mathrm{j} / 2^{\mathrm{i}+1}$ and $\mathrm{j} / 2^{\mathrm{i}}$, respectively. Thus, the conditional probability of a specific proportional occupancy will be,

$\operatorname{prob}\left(\mathrm{O}_{\mathrm{i}+1}=(2 \mathrm{j}-\mathrm{k}) / 2^{\mathrm{i}+1}\right)=\mathrm{C}_{\mathrm{j}}^{\mathrm{k}} \mathrm{p}_{\mathrm{i}+1}^{\mathrm{j}-\mathrm{k}} \mathrm{q}_{\mathrm{i}+1}^{\mathrm{k}}$

where $C_{j}^{k}$ is the binomial coefficient $j ! /(k !(j-k) !)$. Therefore, the recursion relationship for the probability of occupancy $j / 2^{i}$ at the spatial scale $i$ with the grain $A_{0} / 2^{i}$ will be (Hui and McGeoch 2007a),

$\operatorname{prob}\left(\frac{\mathrm{j}}{2^{\mathrm{i}}}\right)=\sum_{\mathrm{k}} \mathrm{C}_{\frac{\mathrm{j}+\mathrm{k}}{2}}^{\mathrm{k}} \mathrm{p}_{\mathrm{i}}^{\frac{\mathrm{j}-\mathrm{k}}{2}} \mathrm{q}_{\mathrm{i}}^{\mathrm{k}} \operatorname{prob}\left(\frac{(\mathrm{j}+\mathrm{k}) / 2}{2^{\mathrm{i}-1}}\right)$

where $\mathrm{k}=0,2,4, \ldots, \operatorname{Min}\left[\mathrm{j}, 2^{\mathrm{i}}-\mathrm{j}\right]$ if $\mathrm{j}$ is an even number and $k=1,3,5, \ldots, \operatorname{Min}\left[j, 2^{i}-j\right]$ if $j$ is an odd number. This model describes the probability of species occupancy at different spatial scales (such as $\mathrm{A}_{0}$, $\mathrm{A}_{0} / 2, \ldots, \mathrm{A}_{0} / 2^{\mathrm{i}}$, ). Further, the mathematical expectation of occupancy at the spatial scale $\mathrm{i}$ with grain $\mathrm{A}_{0} / 2^{\mathrm{i}}$ will be,

$E\left(O_{i}\right)=\sum_{j=0}^{2^{i}} \frac{j}{2^{i}} \operatorname{prob}\left(\frac{j}{2^{i}}\right)$

which is called the scaling pattern of occupancy (Hui et al. 2006, Hui and McGeoch 2007b), also known as the scale-area curve (Kunin 1998) and the area-ofoccupancy (Hartley and Kunin 2003).

\section{Self-similar assumption}

The distribution of a species is called self-similar (Harte et al. 1999) when the coefficient $p_{i}$ and $q_{i}$ are scale independent, i.e. $\mathrm{p}_{\mathrm{i}}=\mathrm{p}$ and $\mathrm{q}_{\mathrm{i}}=\mathrm{q}$ (where $\mathrm{p}$ is referred to as the saturation coefficient, Hui and McGeoch 2007a). This self-similar distribution has been used to model a number of macroecological relationships, i.e. in which $\mathrm{p}=2 \mathrm{a}-1$ and $\mathrm{q}=2(1-\mathrm{a}) \quad(0.5 \leq \mathrm{a} \leq 1 ; \mathrm{a}$ represents the probability that a present species will be found in at least a specific one of the two resulting rectangles (Harte et al. 1999, 2005, Ostling et al. 2003; criticism of this approach are discussed later).
According to the theories of Minkowski-Bouligand dimensionality and the box-counting or capacity dimensionality (Meakin 1998), the expectation of the fractal dimensionality $\mathrm{E}(\mathrm{D})$ is:

$$
\begin{aligned}
E(D) & =E\left(\lim _{\mathrm{i} \rightarrow \infty} \frac{2 \times \ln j(\mathrm{i})}{\mathrm{i} \times \ln 2}\right) \\
& =\lim _{\mathrm{i} \rightarrow \infty} \frac{2 \times \ln \left[\mathrm{j}_{0} \times \prod_{\mathrm{k}=1}^{\mathrm{i}}\left(1+\mathrm{p}_{\mathrm{k}}\right)\right]}{\mathrm{i} \times \ln 2}
\end{aligned}
$$

where $j(i)$ is the number of occupied patches after the $i$ subdivision and $\mathrm{j}_{0}$ is the original number of occupied grids. Under Harte's assumption of self-similarity,

$\mathrm{E}(\mathrm{D})=\frac{2 \times \ln [1+\mathrm{p}]}{\ln 2}=2+\frac{2 \times \ln \mathrm{a}}{\ln 2}$

in which $0 \leq \mathrm{E}(\mathrm{D}) \leq 2$. This implies that the species distribution is fractal under the self-similar distribution.

\section{Power-scaling assumption}

As discussed above, the general assumption of selfsimilarity in species distributions has been criticized, at least partly because species tend to have self-similar distributions only over particular ranges of spatial scales (Lennon et al. 2002, Halley et al. 2004; the criticisms of Maddux 2004 are considered later). The assumption of self-similarity will therefore only hold for a particular species over some spatial scales, and the relationship between scale range and self-similarity will also vary between species. The self-similarity thus varies both within and across species (Lennon et al. 2002).

Here we illustrate the relative scale dependence of Harte's a for a single species using a dataset of subAntarctic cushion plants Azorella selago (Apiaceae) $\left(46^{\circ} 55^{\prime} \mathrm{S}, 37^{\circ} 45^{\prime} \mathrm{E}\right.$; Prince Edward Islands) (Le Roux et al. 2005). We use the above bisection approach to calculate Harte's a for 200 individual plants in a quadrat (black rectangular in Fig. 1A; $A_{0}=441824 \mathrm{~m}^{2}$ ). We used point data, i.e. using the central location of each cushion plant such that each individual exclusively occupies a unique location (as in Williamson's (2003) grid-point system). When the number of bisection times, i, is smaller than 3 or larger than 8, Harte's aremains constant ( $=1$ or 0.5 ). When $\mathrm{i}$ is between 3 and 8 . Harte's a decreases with $\mathrm{i}$ as a power function $(\mathrm{a}=$ $2.2405 \mathrm{i}^{-0.7278}, \mathrm{R}^{2}=0.9942, \mathrm{p}<0.001$ ) (Fig. 1B). This threshold phenomenon happens when the patch size is between $A_{3}=55.23 \mathrm{~m}^{2}$ and $A_{8}=1.726 \mathrm{~m}^{2}$, within which Harte's a is highly linearly correlated with the width of the bisections $\mathrm{w}(\mathrm{a}=0.0991 \mathrm{w}+$ $0.3857, \quad 1.105 \mathrm{~m} \leq \mathrm{w} \leq 6.25 \mathrm{~m}, \quad \mathrm{R}^{2}=0.9955, \quad \mathrm{p}<$ $0.001)$. This result for the distribution of $A$. selago 
A

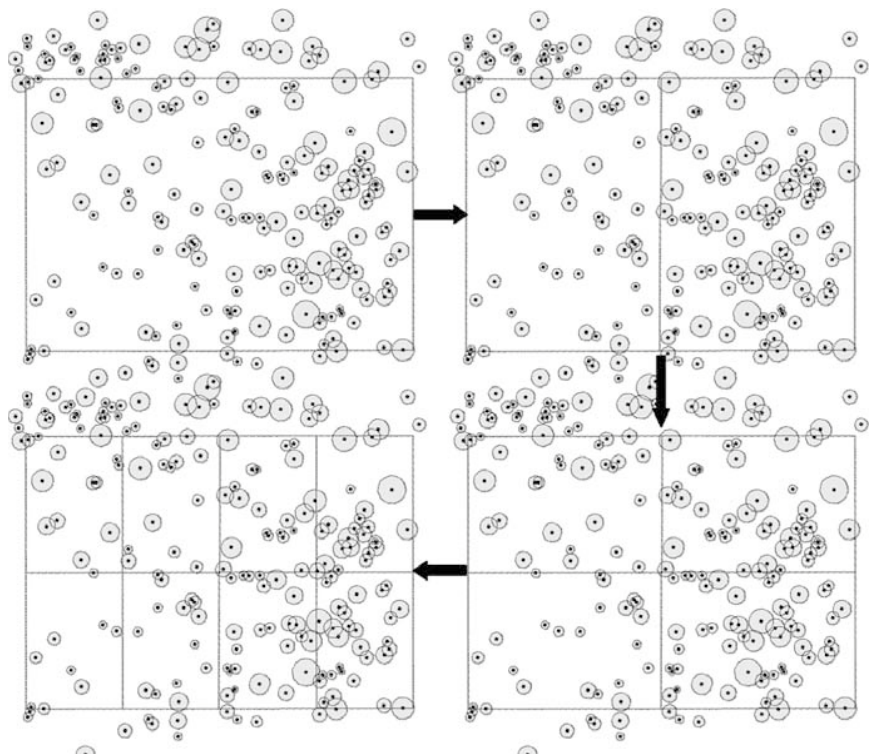

$\odot$

$\odot$
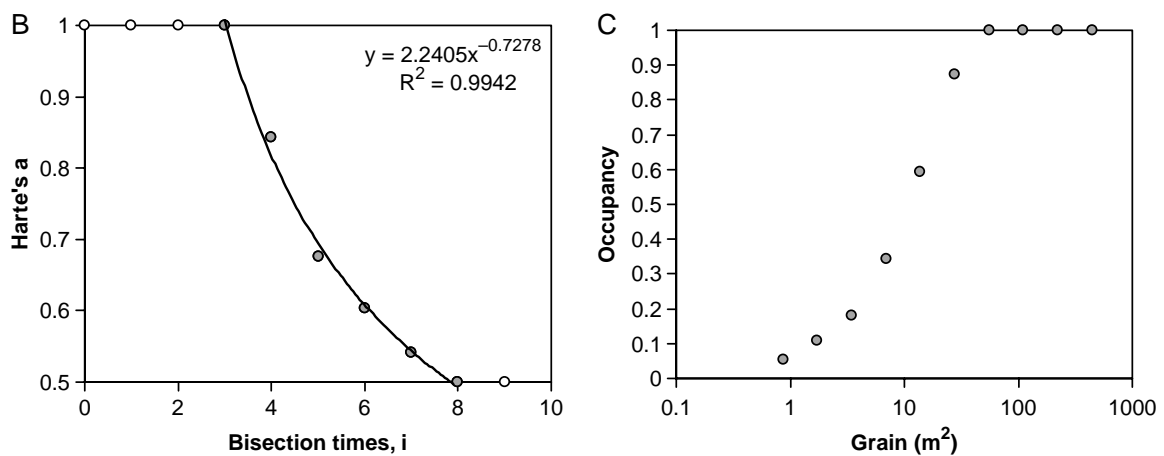

Fig. 1. (A) Spatial distribution of cushion plants (Azorella selago) in a plot on Marion Island. Arrows indicate increasing numbers of bisections. (B) The relationship between Harte's and bisection time. (C) The relationship between sample grain and occupancy.

shows that with increasing grain size, occupancy follows a general percolation process (Fig. 1C), i.e. with the increase in grain, occupancy at first increases slowly, accelerates and eventually plateaus towards maximum occupancy (Fig. 1C) (He and Hubbell 2003, Hui et al. 2006, Hui and McGeoch 2007b).

Based on the above finding, we therefore assume $\mathrm{a}_{\mathrm{i}}=\mathrm{c} \times \mathrm{i}^{-\mathrm{d}}$, in which $\mathrm{c}$ denotes the grain at which Harte's fractal assumption begins to decay and $\mathrm{d}$ determines the speed of decay from high to low dimensions; and therefore we have,

$\mathrm{E}(\mathrm{D})=\lim _{\mathrm{i} \rightarrow \infty}\left(2+\frac{2 \times \operatorname{lnc}}{\ln 2}-\frac{2 \mathrm{~d} \times \ln (\mathrm{i} !)}{\mathrm{i} \times \ln 2}\right)$

The value of $E(D)$ changes from 2 to 0 as the number of bisection times, i, increases. Therefore, it is inappropriate to use Harte's assumption of self-similarity to describe species distributions and community structure over the intermediate range of grain sizes, because the self-similarity in the species distribution is broken. Rather, varying values of Harte's a and of the saturation coefficient $\mathrm{p}_{\mathrm{i}}$ should be adopted over this range.

We now compare the outcome of the OPT model under the self-similar assumption and under the above power-scaling assumption. We use a stochastic probability simulation in a two-dimensional landscape. The landscape is bisected according to Eq. 1 the probability decided by the self-similarity rule (p, q) and Eq. 2 the scale-dependent rule $\left(\mathrm{p}_{\mathrm{i}}, \mathrm{q}_{\mathrm{i}}\right)$,

$\mathrm{p}_{\mathrm{i}}= \begin{cases}1, & \text { if } \mathrm{i} \leq \mathrm{c}^{1 / \mathrm{d}} \\ 2 \mathrm{c} \times \mathrm{i}^{-\mathrm{d}}-1, & \text { if } \mathrm{c}^{1 / \mathrm{d}} \leq \mathrm{i} \leq(2 \mathrm{c})^{1 / \mathrm{d}} \\ 0, & \text { if } \mathrm{i} \geq(2 \mathrm{c})^{1 / \mathrm{d}}\end{cases}$

Note that here the two critical thresholds $\left(c^{1 / d}\right.$ and $\left.(2 \mathrm{c})^{1 / d}\right)$ for the bisection times $\mathrm{i}$ that determine the range within which the power-function is present. These limits are determined by the maximum and 
minimum dimensionality of a 2-dimensional object (Mandelbrot 1983), as well as the only two consistent coefficients of Harte's a $(a=1$, or $1 / 2$; Appendix D in Maddux 2004). Although setting these thresholds may result in incorrect estimation of occupancy at extreme scales, this approach is commonly used and the effect generally taken to be insignificant (Grossi et al. 2001).

We use Mathematica 5.0 to build this simulated stochastic probability simulation to explicitly illustrate and compare the distribution patterns, scaling pattern of occupancy, as well as the number of occupied patches at different bisection times arising from the two approaches. The simulation input that was used for the self-similar distribution was $\mathrm{a}(\mathrm{a}=(1+\mathrm{p}) / 2)$ and a is thus different for each species. The input used for the power-scaling distribution was $p_{i}$ according to Eq. 7 . The probability distribution of occupancy (Eq. 2), i.e. the probability that a particular value of occupancy is generated, will also be compared for these two assumptions (self-similar and power-scaling) to test the scale dependence and parameter sensitivity of the models.

\section{Generation of interspecific occupancy frequency distributions}

Because each species is likely to have a comparatively unique occupancy scaling pattern, we superimposed the occupancy probability distribution generated for individual 'species', as outlined above, to generate an interspecific occupancy frequency distribution, i.e. for an assemblage of species. Hui and McGeoch (2007a) adopted this approach to model interspecific occupancy frequency distributions. We assume that in a community every species has unique parameters: Harte's a for the self-similar assumption of species distribution and parameters $\mathrm{c}$ and $\mathrm{d}$ for the power-scaling assumption. By combining or superimposing the species probability distributions obtained above, we obtain a community level (interspecific) occupancy frequency distribution. Because there are no previous community-level estimates in the literature of the above parameters, we selected the parameters randomly from their range of possible values (a similar method has been used by Lennon et al. 2002). Communities ranging in size from 10 to 1000 species were used to generate occupancy frequency distributions. Because community richness was found to have a negligible effect on the shape of the distribution (results not shown here; see also Hui and McGeoch 2007a) we report results only for a community of 100 species. Hanski's (1982) core and satellite terminology (referring to the last and first classes of the occupancy distribution and the species occurring in them) are adopted for convenience to describe the shapes of occupancy frequency distributions (see also McGeoch and Gaston 2002).

\section{Results}

The spatial distribution of individuals generated by the two dimensional stochastic probability simulation under the self-similar assumption is obviously different from that obtained under the power-scaling assumption (Fig. 2A-B). The self-similar distribution is, as expected for a typical Cantor-set fractal structure (e.g. Menger's 1928 sponge and Sierpinski's 1915 carpet), more highly spatially aggregated (Fig. 2A). By contrast, individuals from the power-scaling distribution are comparatively dispersed (Fig. 2B), more closely resembling the empirical distribution of cushion plants (Fig. 1A). Under the self-similar assumption, the scaling pattern of occupancy was log-log linear (Fig. 2C), whereas it was nonlinear and logistic under the powerscaling assumption (Fig. 2D).

The log-log linear scaling pattern of occupancy that emerged (Fig. 2C) is a consequence of the expected value of occupancy $\mathrm{E}\left(\mathrm{O}_{\mathrm{i}+1}\right)=(\mathrm{p}+0.5 \mathrm{q}) \mathrm{E}\left(\mathrm{O}_{\mathrm{i}}\right)=\mathrm{a} \times$ $\mathrm{E}\left(\mathrm{O}_{\mathrm{i}}\right)$, we have $\mathrm{E}\left(\mathrm{O}_{\mathrm{i}}\right)=\mathrm{a}^{\mathrm{i}}$ and the number of occupied patches $\mathrm{N}_{\mathrm{i}}=2^{\mathrm{i}} \mathrm{E}\left(\mathrm{O}_{\mathrm{i}}\right)=(2 \mathrm{a})^{\mathrm{i}}$ (noted that $2 \mathrm{a}>1$ ). This is a power relationship of occupancy $\mathrm{E}\left(\mathrm{O}_{\mathrm{A}}\right)=\delta \times$ $\mathrm{A}^{-\log _{2} \mathrm{a}}$, where $\delta$ is a constant and $1 \geq-\log _{2} \mathrm{a} \geq 0$. Because sample grain a can not be larger than sample extent (the maximum size of the landscape), the curve generated from the self-similar distribution in Fig. 2C will not reach saturation (level off), in contrast with the curve generated by the power-scaling assumption (Fig. 2D). Under the self-similar assumption, the number of occupied patches shows no upper limit (Fig. 2E), whereas the number of occupied patches reached an asymptote under the power-scaling assumption (Fig. 2F).

According to Eq. 2, we find also that the effect of the number of bisections (that determines sample grain) on the occupancy probability distribution under the selfsimilarity assumption differs markedly from the distribution under the power-scaling assumption (Fig. 3). The probability distributions under both assumptions become more skewed to the right with the increase in bisection number (i.e. with a decrease in grain), albeit more markedly so under the power-scaling assumption (Fig. 3B). Under both assumptions, when the bisection number (i) is low (e.g. $\mathrm{i}=3$ in Fig. 3), the probability of species being in the core mode (high occupancy) is very high and the distribution is in fact left-skewed (Fig. 3A). With an increase in bisection number, the probability associated with the core mode decreases rapidly, which finally leads to an approximately amodal distribution under the self-similarity assumption (Fig. 3A). However, under the power-scaling assumption, the mode of 

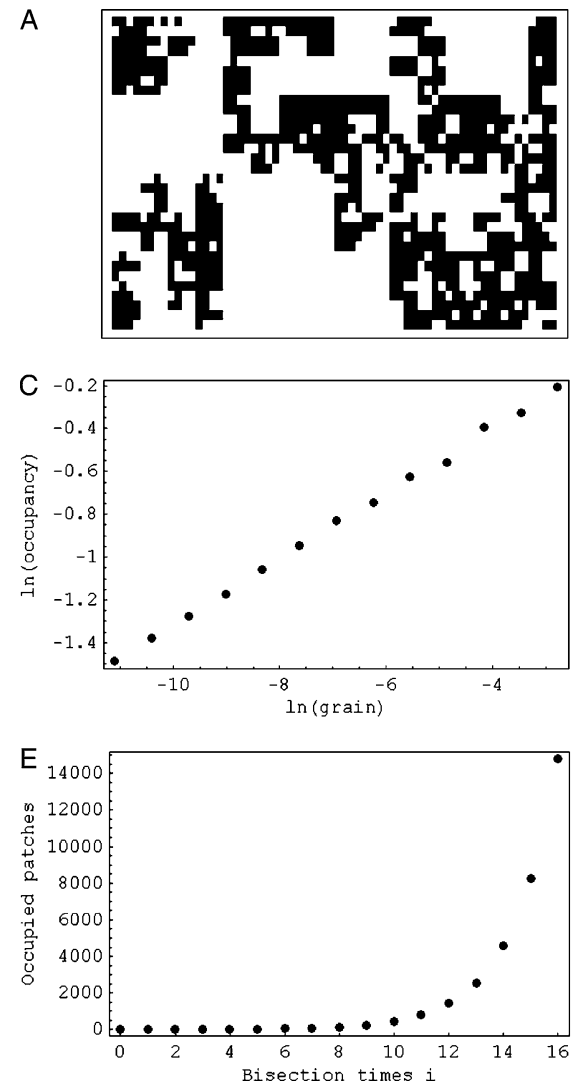

B
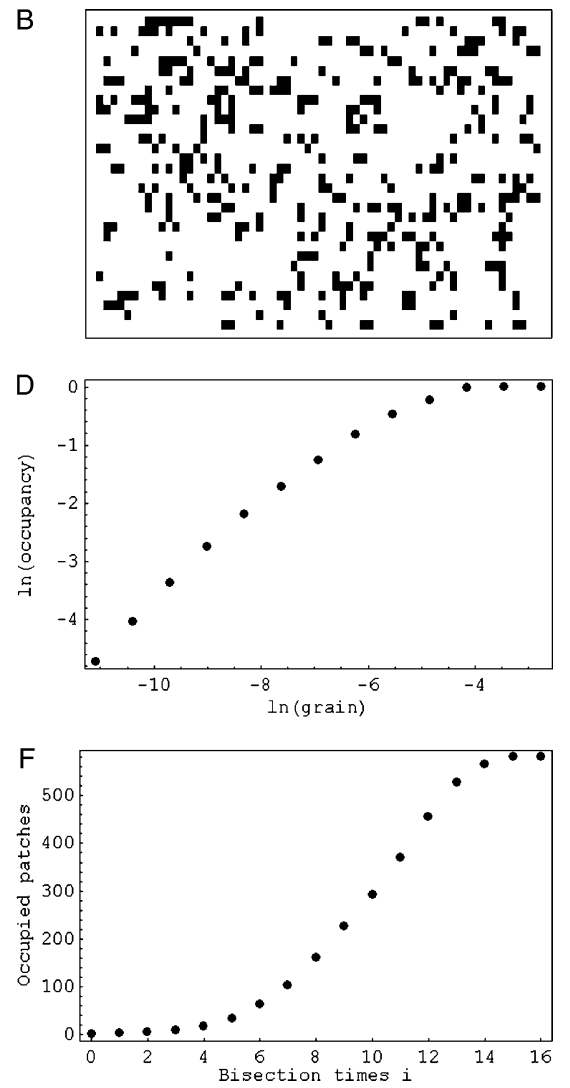

Fig. 2. Simulation results of the spatial distribution of cushion plants (A and B), relationships between occupancy and grain (C and $\mathrm{D}$ ), and the number of occupied patches (E and F). Left panel is under the self-similarity assumption; right panel is under the power-scaling assumption. Parameter values: $\mathrm{a}=0.9 ; \mathrm{c}=2.4 ; \mathrm{d}=0.58 ; \mathrm{A}_{0}=1$; grain $=2^{-12}$ for $\mathrm{A}$ and $\mathrm{B}$.

the probability distribution rapidly moves to the left with the increase in bisection number (i) (Fig. 3B). Grain size therefore alters the shape of occupancy probability distributions in very different ways under self-similar vs power-scaling assumptions. Whereas the greatest modal probability shifts clearly from high to low occupancy under the power-scaling assumption (Fig. 3B), the modal position shifts comparatively little under the self-similar assumption and remains above an occupancy of 0.6 (Fig. 3A).

The value of Harte's a (the self-similarity coefficient) also has an important influence on the shape of the probability distribution. The position of the mode shifts from an occupancy of approximately 0.2 to 0.8 with a change in Harte's a from 0.6 to 0.9 (Fig. 4A). The distributions derived from the OPT model show that the probability distribution of occupancy becomes apparently multimodal when this self-similarity coefficient is larger than a particular threshold (e.g. with $\mathrm{a}=$ 0.975 in Fig. 4A) and leads to discontinuities in the probability distribution (Fig. 4A insert). In contrast, when the self-similarity assumption is broken, the probability distribution becomes smoothly unimodal
(Fig. 4B). Furthermore, an increase in coefficient $\mathrm{c}$ dramatically shifts the mode of the probability distribution to the right (high occupancy). A decrease in coefficient $\mathrm{d}$ has a similar effect, i.e. shifts the mode of the distribution to the right (with the value of $\mathrm{c}$ held constant) (Fig. 4B).

Two general characteristics of the probability distribution generated from the OPT model are worth noting. (1) As in all probability distributions, the area under the lines in Fig. 4 equals one, i.e. all the curves of probability distribution have same underlying area. This is the basic rule of any probability distribution, i.e. the sum of all the probability of all the possible events equals one, hereafter called the unity rule. (2) When the modal position is central (intermediate occupancy) it tends to be broader than when the mode is either at high or low occupancies (Fig. 4), i.e. the width of the mode of the probability distribution is greater when that mode has a central occupancy value, and it is narrower near the occupancy boundaries $(0$ and 1 are the minimal and maximal boundaries of occupancy). The modal probability is thus low at intermediate occupancies and high towards the 

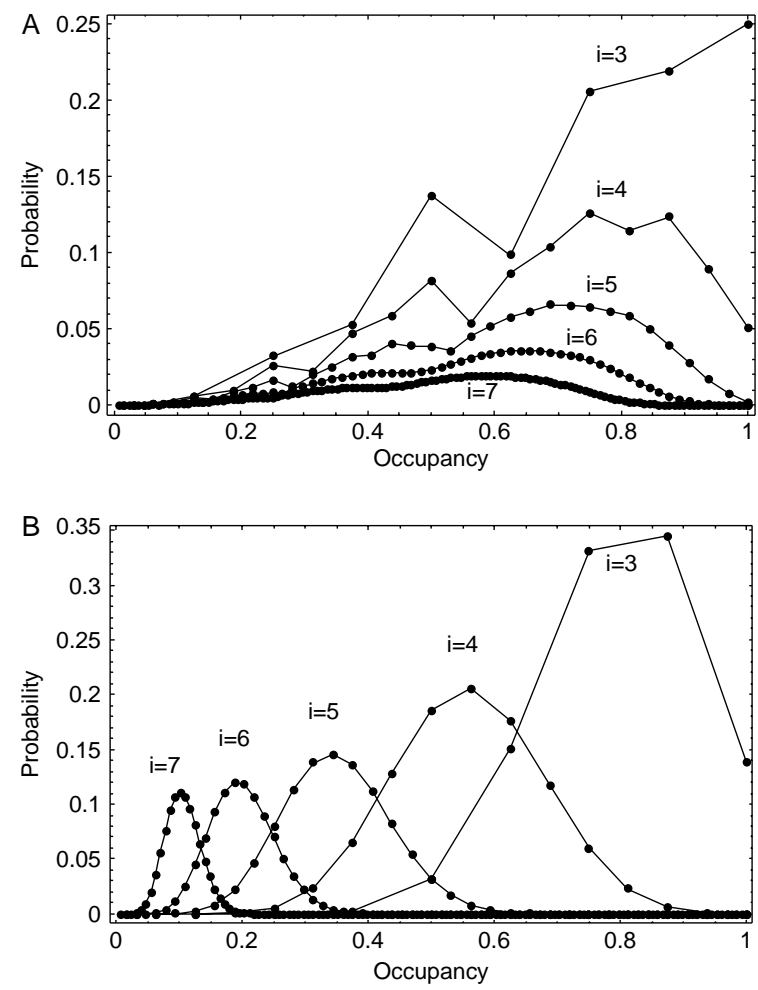

Fig. 3. The effect of the number of bisections (i, related to sample grain) on the probability distribution of occupancy (A) under the self-similarity assumption and (B) under the powerscaling assumption. Parameter values: $\mathrm{p}=0.82 ; \mathrm{c}=1.4$; $\mathrm{d}=0.5$.

occupancy boundaries, hereafter called the shape rule. The simulation results (Fig. 5) show that bimodality in the occupancy frequency distribution at the community level is a direct result of the shape rule of the probability distribution as outlined above (Fig. 4). Bimodality in interspecific occupancy frequency distributions is thus generated directly from the OPT model as a consequence of these statistical properties of occupancy probability distributions.

Although the effect of sample grain on the probability distribution is different under the self-similarity assumption compared to under the power-scaling assumption (Fig. 3), the emergent community level occupancy frequency distributions under the two assumptions are similar (compare Fig. 5A with 5B, 5C and 5D). This shows that the predictions of the OPT model for the shape of occupancy frequency distributions are relatively insensitive to the assumptions of species distribution (either self-similar or power-scaling).

In addition, regardless of the assumption, with an increase in grain (scaling up) the frequency of satellite species (low occupancy) decreases, the frequency of the
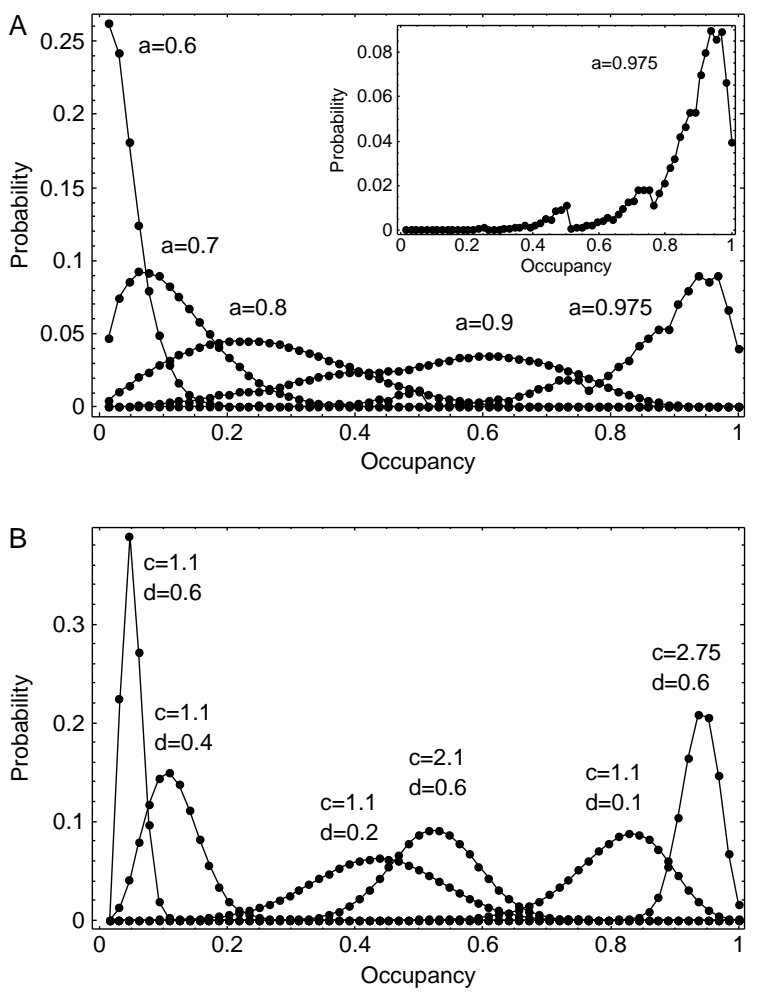

Fig. 4. The effect of model parameters on the probability distribution of occupancy (A) under the self-similarity assumption and (B) under the power-scaling assumption $(i=6)$.

core species (high occupancy) increases, and the frequency of the intermediate occupancy classes changes slightly or remains constant (Fig. 5). This result predicts that the occupancy frequency distribution changes from a satellite-dominated bimodal to a core-dominated bimodal with scaling-up, a prediction supported by empirical studies (McGeoch and Gaston 2002).

\section{Discussion}

The OPT model used here is a species-based model, where each species has its own scaling parameters (a, c and d). The bimodality in the occupancy frequency distribution that is produced under either of the two assumptions used (self-similar or power-scaling) is a statistical outcome of the shape of the species occupancy probability distribution (i.e. the unity and shape rules mentioned above) (Hui and McGeoch 2007a). Moreover, with the increase in spatial scale (sample grain), the shape shifts from satellite-dominated to coredominated bimodalities. These predictions from the 


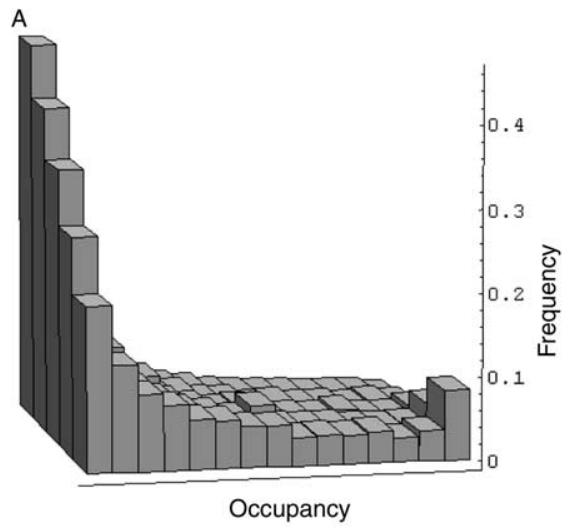

C

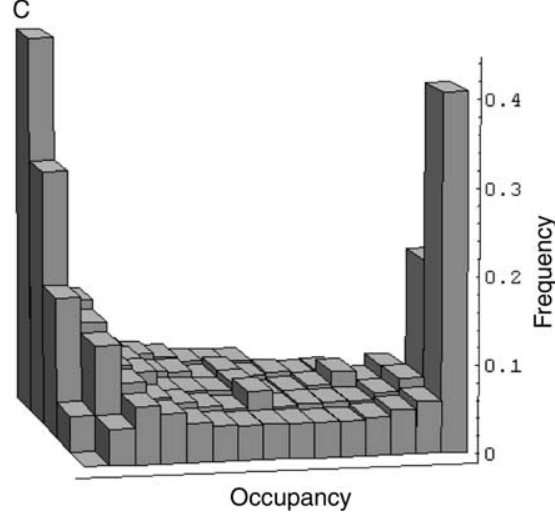

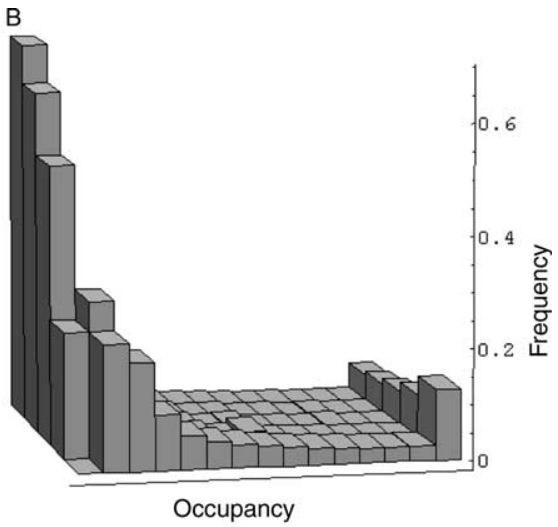

D

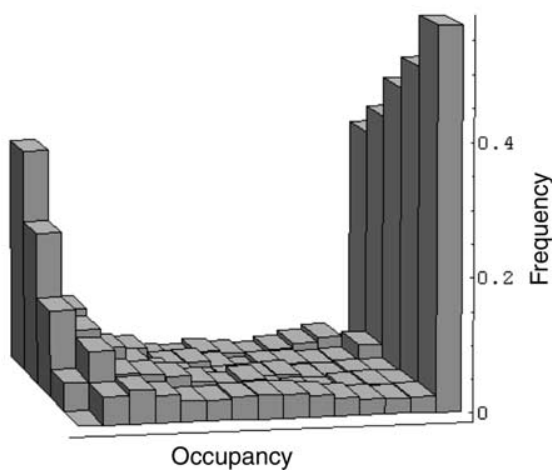

Fig. 5. Simulated interspecific occupancy frequency distributions (A) under the self-similar assumption and (B, C and D) under the power-scaling assumption. The distribution on the $\mathrm{z}$ axis, from the front to the back, is under increasing number of bisections ( $i=4,5, \ldots, 8)$, or decreasing grain. A combination of 100 probability distributions was superimposed to generate these occupancy frequency distributions. (A) The parameter $\mathrm{p}$ is randomly chosen from the range $(0,1)$. (B) The parameter $d$ is randomly chosen from the range $(0,1)$ and $c=1.1$. (C) The parameter $c$ is randomly chosen from the range (1-3) and . (D) The parameters $\mathrm{c}$ and $\mathrm{d}$ are both randomly chosen from the range (1-3) and (0-1) respectively.

model, under either self-similar or power-scaling assumptions, fit the results of several empirical studies (Blackburn and Gaston 1998, Cowley et al. 1999, Guo et al. 2000, McGeoch and Gaston 2002, Storch and Šizling 2002). An increase in grain has been shown to result in a displacement of the species in an assemblage from the left (low proportional occupancy) towards the right (high proportional occupancy) of the occupancy distribution (Fig. 3 in McGeoch and Gaston 2002). The fact that community-level properties are insensitive to the use of the self-similar species distribution has been shown for other patterns (Lennon et al. 2002, Šizling and Storch 2004).

Although Harte's bisection framework with a selfsimilar assumption has received much positive attention (Harte et al. 2001, 2005, Lennon et al. 2002, Šizling and Storch 2004, Conlisk et al. 2007), it has also been criticized (Maddux and Athreya 1999, Maddux 2004, Pueyo 2006). Maddux's (2004) criticism of Harte et al. (1999) (and Ostling et al. 2000, Plotkin et al. 2000) is based on the demonstration that the probability rule- based distribution of species in a landscape (i.e. a constant a) divided into well-shaped units (derived from Harte et al.' 1999 bisection scheme) produces biologically unrealistic estimates of species richness. As a result the probability rule is considered to be inappropriate for relating community level patterns to selfsimilar species distributions (Lennon et al. 2002). This criticism is not of the bisection scheme (and the resulting shape of sample units) per se, but of the use of the probability rule (in which Harte's a is constant) to generate species distributions across the landscape that has been subdivided using the bisection scheme (as will be demonstrated elsewhere). Maddux's (2004) criticisms, however, do not hold when the self-similarity assumption is broken (i.e. when Harte's $\mathrm{a}$ is not constant). For example, the key Eq. $7(\mathrm{~L}(\mathrm{x}, \mathrm{o}, \mathrm{o})=$, $\mathrm{L}(\mathrm{o}, \mathrm{o}, \mathrm{x})=(2 \mathrm{a}-1)(1-\mathrm{a}))$ in Maddux (2004) no longer stands when the distributions are no longer self-similar (as evident from $\mathrm{L}(\mathrm{x}, \mathrm{o}, \mathrm{o})=\left(2 \mathrm{a}_{1}-1\right)\left(1-\mathrm{a}_{2}\right) ; \mathrm{L}(\mathrm{o}, \mathrm{o}, \mathrm{x})=$ $\left.\left(1-\mathrm{a}_{1}\right)\left(2 \mathrm{a}_{2}-1\right) ; \mathrm{L}(\mathrm{x}, \mathrm{o}, \mathrm{o}) \neq \mathrm{L}(\mathrm{o}, \mathrm{o}, \mathrm{x})\right)$. This argument holds for rectangular sampling units, but also applies to 
square shaped sample units. Therefore, the OPT model with a power-scaling assumption does not fall foul of Maddux (2004).

The OPT model with a self-similarity assumption did indeed produce biologically less realistic patterns under particular conditions. The reason for this is as follows. In the occupancy probability distribution, a single species may approach different probabilities of having the same occupancy (occupancy around 0.5 when $\mathrm{a}=0.975$, Fig. $4 \mathrm{~A}$ ). This result arises as a consequence of the discontinuities that occur in the occupancy probability distribution under the selfsimilar assumption at high values of Harte's a (Fig. 4A). However, the same problem does not occur when the self-similarity assumption is broken (Fig. 4B). This result demonstrates that a self-similarity assumption is not appropriate for modeling species distributions, particularly at fine scales (such as for stands of cushion plants on Marion Island).

Nevertheless, we demonstrate here that although species level occupancy properties generated by the selfsimilarity assumption may be uncommon, at a community level the outcome of the two assumptions are largely indistinguishable. In other words, both assumptions lead to empirically realistic interspecific occupancy frequency distributions, with the probability and size of the core mode increasing with an increase in sample grain (McGeoch and Gaston 2002). Indeed, Harte et al. (2001) pointed out that self-similarity at the species level is generally incompatible with self similarity at the community level. Here we obtained a $\log$-log linear scaling pattern of occupancy at the species level using the self-similarity assumption. Kunin (1998) also found a $\log$-log linear relationship between occupancy and sample grain, and this is in fact a result of the adoption of the self-similar assumption (as we show in Fig. 2C) (Kunin 1998, Kunin et al. 2000). However, it has been demonstrated that a non-linear, logistic scaling pattern of occupancy is more frequently observed in nature $(\mathrm{He}$ and Gaston 2000, Hartley and Kunin 2003, Gaston et al. 2006). Using the power-scaling assumption we were also able to produce such a non-linear, logistic scaling pattern of occupancy (Fig. 2D). This logistic shape of the scaling pattern of occupancy concurs with previous work (He and Gaston 2003, Hui et al. 2006, Gaston et al. 2006), indicating a nonlinear coalition process of occupied patches with the increase in grain (i.e. a percolation process) (Hui and McGeoch 2007b). Therefore, although the community level properties that emerge from the alternative assumptions (selfsimilar versus power-scaling) are similar, the species level properties used to generate the interspecific occupancy frequency distribution are more biologically realistic under the power-scaling assumption. Impor- tantly, therefore, it appears that the shape of occupancy frequency distributions comparatively uninformative with regards to the generating processes at the species level.

Therefore, here we show that the application of the OPT model with a power-scaling assumption of species distributions provides a robust framework for modeling interspecific distribution patterns. We show that at relatively fine spatial scales (such as across individual stands of plants), the self-similarity of species distributions decay, clearly following a power relationship $\mathrm{a}_{\mathrm{i}}=\mathrm{c} \times \mathrm{i}^{-\mathrm{d}}$. This result proposes two scale-independent parameters (i.e. $\mathrm{c}$ and $\mathrm{d}$ ) for describing the pattern of decay of species distributions from high to low fractal dimensionality. This approach provides a model for understanding the mechanisms generating species distribution patterns at both species and community levels. However, in agreement with Hui and McGeoch (2007a), the OPT model under the power-scaling assumption demonstrates that the empirical patterns observed in interspecific occupancy frequency distributions are largely a consequence of the statistical properties of occupancy probability distributions (the unity and shape rules). Further studies are required to examine if, and if so the ways in which, empirical occupancy frequency distributions differ from the general pattern generated by the statistical properties of the occupancy probability distribution.

The two assumptions regarding species distributions lead to relatively similar patterns of interspecific occupancy frequency distributions. However, the spatial distribution of individual species and the scaling pattern of occupancy differ distinctly between the two assumptions. These results also emphasize the importance of caution when comparing empirical results with model outcomes (Levin 1992). A validity of a model can not be tested based only on its match with empirical patterns because different models (mechanisms) can produce similar spatiotemporal patterns. The acceptance of a model based solely on its fit to empirical data is thus likely to suffer from high type II error rates. The validity of an ecological model can only be tested by decomposing it into its component parts to test the individual relationships and processes from which the model is constructed. Therefore, by virtue of the biological realism of the occupancy scaling patterns produced, our results suggest that the use of the bisection method in combination with a power-scaling assumption is more appropriate for modeling species distributions than the use of a self-similarity assumption. 
Acknowledgements - We are grateful to Annette Ostling, Peter C. Le Roux, Nicholas Gotelli and Arnost L. Šizling for comments, and the DST-NRF Centre of Invasion Biology for financial support.

\section{References}

Blackburn, T. M. and Gaston, K. J. 1997. Who is rare? Artefacts and complexities of rarity determination. - In: Kunin, W. E. and Gaston, K. J. (eds), The biology of rarity. Chapman and Hall, pp. 48-58.

Conlisk, E. et al. 2007. A new class of models of spatial distribution. - Ecol. Monogr. 77: 269-284.

Cowley, M. J. R. et al. 1999. Flight areas of British butterflies: assessing species status and decline. - Proc. R. Soc. Lond. B 266: 1587-1592.

Gaston, K. J. 1994. Rarity. - Chapman and Hall.

Gaston, K. J. 1996. Species-range-size distributions: patterns, mechanisms and implications. - Trends Ecol. Evol. 11: 197-201.

Gaston, K. J. and Blackburn, T. M. 2000. Pattern and process in macroecology. - Blackwell.

Gaston, K. J. et al. 2006. Abundance, spatial variance and occupancy: arthropod species distribution in the Azores. - J. Anim. Ecol. 75: 646-656.

Grossi, L. et al. 2001. Statistical detection of multiscale landscape patterns. - Environ. Ecol. Stat. 8: 253-267.

Guo, Q. et al. 2000. Abundance and distribution of desert animals: are spatial and temporal patterns related? - J. Ecol. 88: 551-560.

Halley, J. M. et al. 2004. Uses and abuses of fractal methodology in ecology. - Ecol. Lett. 7: 254-271.

Hanski, I. 1982. Dynamics of regional distribution: the core and satellite species hypothesis. - Oikos 38: 210-221.

Hanski, I. and Gyllenberg, M. 1993. Two general metapopulation models and the core-satellite species hypothesis. Am. Nat. 142: 17-41.

Harte, J. and Kinzig, A. P. 1997. On the implications of species-area relationships for endemism, spatial turnover, and food web patterns. - Oikos 80: 417-427.

Harte, J. et al. 1999. Self-similarity in the distribution and abundance of species. - Science 284: 334-346.

Harte, J. et al. 2001. Self similarity and the relationship between abundance and range size. - Am. Nat. 157: 374386.

Harte, J. et al. 2005. A theory of spatial structure in ecological communities at multiple spatial scales. - Ecol. Monogr. 75: 179-197.

Hartley, S. and Kunin, W. E. 2003. Scale dependence of rarity, extinction risk, and conservation priority. - Conserv. Biol. 17: 1559-1570.

He, F. and Gaston, K. J. 2000. Estimating species abundance from occurrence. - Am. Nat. 156: 553-559.

He, F. and Legendre, P. 2002. Species diversity patterns derived from species-area models. - Ecology 83: 11851198.

He, F. and Gaston, K. J. 2003. Occupancy, spatial variance, and the abundance of species. - Am. Nat. 162: 366-375.
He, F. and Hubbell, S. P. 2003. Percolation theory for the distribution and abundance of species. - Phys. Rev. Lett. 91: 198103.

Hui, C. and McGeoch, M. A. 2007a. A self-similarity model for the occupancy frequency distribution. - Theor. Popul. Biol. 71: 61-70.

Hui, C. and McGeoch, M. A. 2007b. Capturing the 'droopy tail' in the occupancy-abundance relationship. - Écoscience 14: 103-108.

Hui, C. et al. 2006. A spatially explicit approach to estimating species occupancy and spatial correlation. - J. Anim. Ecol. 75: $140-147$.

Kunin, W. E. 1998. Extrapolating species abundance across spatial scales. - Science 281: 1513-1515.

Kunin, W. E. et al. 2000. Scaling down: on the challenge of estimating abundance from occurrence patterns. - Am. Nat. 156: 560-566.

Le Roux, P. C. et al. 2005. Effects of a short-term climate change experiment on a sub-Antarctic keystone plant species. - Global Change Biol. 11: 1-12.

Lennon, J. J. et al. 2002. Fractal species distributions do not produce power-law species-area relationships. - Oikos 97: 378-386.

Levin, S. A. 1992. The problem of pattern and scale in ecology. - Ecology 73: 1943-1967.

Maddux, R. D. 2004. Self-similarity and the species-area relationship. - Am. Nat. 163: 616-626.

Maddux, R. D. and Athreya, K. 1999. On the distribution and abundance of species. - Science 286: 1647a.

Mandelbrot, B. B. 1983. The fractal geometry of nature. - Freeman.

Menger, K. 1928. Dimensionstheorie. - B.G. Teubner Publishers.

McGeoch, M. A. and Gaston, K. J. 2002. Occupancy frequency distributions: patterns, artefacts and mechanisms. - Biol. Rev. 77: 311-331.

Meakin, P. 1998. Fractals, scaling and growth far from equilibrium. - Cambridge Univ. Press.

Nee, S. et al. 1991. Core and satellite species: theory and artefacts. - Oikos 62: 83-87.

Ostling, A. et al. 2003. A community-level fractal property produces power-law species-area relationships. - Oikos 103: $218-224$.

Ostling, A. et al. 2004. Self-similarity, the power-law form of the species-area relationship, and a probability rule: a reply to Maddux. - Am. Nat. 163: 627-633.

Papp, L. and Izsák, J. 1997. Bimodality in occurrence classes: a direct consequence of lognormal or logarithmic series distribution of abundances - a numerical experimentation. - Oikos 79: 191-194.

Plotkin, J. et al. 2000. Species-area curves, spatial aggregation, and habitat specialization in tropical forests. - J. Theor. Biol. 207: 81-99.

Pueyo, S. 2006. Self-similarity in species-area relationship and in species abundance distribution. - Oikos 112: 156-162.

Scheiner, S. M. and Rey-Benayas, J. M. 1997. Placing empirical limits on metapopulation models for terrestrial plants. - Evol. Ecol. 11: 275-288. 
Sierpiński, W. 1915. Su rune courbe dont tout point est une point de ramification. - C. R. Acad. Sci. Paris 160: 302305.

Šizling, A. L. and Storch, D. 2004. Power-law species-area relationships and self-similar species distributions within finite areas. - Ecol. Lett. 7: 60-68.

Storch, D. and Sizling, A. L. 2002. Patterns of commonness and rarity in central European birds: reliability of the coresatellite hypothesis within a large scale. - Ecography 25: 405-416.
Tokeshi, M. 1992. Dynamics of distribution in animal communities: theory and analysis. - Res. Popul. Ecol. 34: 249-273.

Van Rensburg, B. J. et al. 2000. Testing generalities in the shape of patch occupancy frequency distributions. Ecology 81: 3163-3177.

Wiens, J. A. 1989. Spatial scaling in ecology. - Funct. Ecol. 3: 385-397.

Williamson, M. 2003. Species-area relationships at small scales in continuum vegetation. - J. Ecol. 91: 904-907. 